\title{
FOLFIRINOX: a new standard of care in the adjuvant setting of resectable pancreatic adenocarcinomas
}

\author{
Abdel Hadi Hajjar ${ }^{1}$, Roland Eid ${ }^{1}$, Fady Gh Haddad ${ }^{1}$ \& Hampig Raphael Kourie*,1 \\ ${ }^{1}$ Hematology \& Oncology Department, Faculty of Medicine, Saint Joseph University, Beirut, Lebanon \\ *Author for correspondence: hampig.kourie@hotmail.com
}

“FOLFIRINOX might be the protocol of choice in the adjuvant setting in resectable pancreatic cancer. However, the choice of chemotherapy regimen should be based on tumor and patients' characteristics such as the stage of the disease, age, comorbidities and performance status."

First draft submitted: 11 March 2019; Accepted for publication: 22 March 2019; Published online: 12 June 2019

Pancreatic cancer is among the most aggressive solid tumors, representing the fourth most common cause of death by cancer in men and women worldwide [1]. The 5-year overall survival is 5\%; but it becomes $2 \%$ in the case of metastatic disease [2]. Those low survival rates are partially due to the late diagnosis of this disease. Indeed, $80 \%$ of pancreatic cancers are diagnosed at locally advanced or metastatic stages. Thus, only $20 \%$ of cases can be treated in a 'curative intent' by a multimodality treatment $[2,3]$. Note that the majority of pancreatic cancers are adenocarcinomas.

Many difficulties surround the treatment of pancreatic cancer. In fact, the latter is a genetically complex and heterogeneous tumor that results from many genetic mutations, affecting therefore multiple signaling pathways [4]. Therefore, chemotherapy and targeted therapy might not be an option in pancreatic cancer treatment [5]. Tumor stroma is also a key factor in the resistance to treatment, by limiting the drug delivery to cancerous cells [6].

Surgery remains the cornerstone of the management of early-stage pancreatic cancer. Many trials tried to evaluate the role of different adjuvant treatment regimens going from chemotherapy to radiation therapy, alone or in combination. The first molecule to be approved was gemcitabine based on the results of the CONKO-001 trial conducted on a total of 368 patients. First, it was proven that the use of gemcitabine monotherapy for 6 months prolonged disease-free survival compared with observation alone (13.4 vs 6.9 months) [7]. Second, an improvement in overall survival (OS) was also demonstrated (22.8 vs 20.2 months) [8]. Adjuvant chemotherapy with gemcitabine was well tolerated, and grade 3 or 4 toxicities occurred infrequently. In western countries, and until 2016, the use of gemcitabine monotherapy in the adjuvant setting was the standard of care in pancreatic cancer.

Note that during the gemcitabine monotherapy era, other clinical studies were conducted. One particular study targeted periampullary adenocarcinoma (ESPAC-3) and showed that there was no significant difference in survival with 5-FU/folinic acid compared with gemcitabine (23 vs 23.9 months). Gemcitabine remained the standard of care due to its lesser toxicities compared with 5-FU [9]. Another study conducted in Japan (JASPAC-1) showed that the chemotherapeutic agent S1 (tegafur, a prodrug of 5-FU) showed a better progression-free survival (PFS) compared with the standard gemcitabine (23.2 vs 12.2 months) and a better OS too (not reached vs 25.9 months) alongside a better quality of life. However, those results were favorable in the Japanese population and do not necessarily apply to the Caucasian one. Therefore, despite the fact that S1 became the standard of care in Japan, gemcitabine was still the drug of choice in western countries [10].

Then, in 2016, after considering the gemcitabine as the only adjuvant treatment for years, another alternative saw the light. In fact, the ESPAC-4 study conducted on 732 patients compared gemcitabine monotherapy and the combination gemcitabine/capecitabine. This combination has synergism between the intracellular metabolites of capecitabine and gemcitabine on thymidylate synthase. The combination showed a better OS than monotherapy ( 28 vs 25.5 months) and better 5 -year survival rates (28.8 vs $16.3 \%)$. Even though combination therapy proved to be more efficient, more grade 3 and grade 4 adverse events were noted with this alternative treatment. Consequently,

Future Medicine 
Table 1. Comparison between the three adjuvant chemotherapy regimens in resectable pancreatic cancer.

\begin{tabular}{|c|c|c|c|}
\hline $\begin{array}{l}\text { Patients characteristics, side } \\
\text { effects and outcomes }\end{array}$ & Gemcitabine monotherapy $^{\dagger}$ & Gemcitabine + capecitabine ${ }^{\ddagger}$ & Modified FOLFIRINOX $\$$ \\
\hline Median age & 62 years old & 65 years old & 63 years old \\
\hline Male sex & $59 \%$ & $55 \%$ & $57.5 \%$ \\
\hline Tumor stage & $\begin{array}{l}\text { T1 4\% } \\
\text { T2 10\% T3 82\% } \\
\text { T4 4\% }\end{array}$ & $\begin{array}{l}\text { Stage I } 4 \% \\
\text { Stage II } 5 \% \\
\text { Stage III } 90 \% \\
\text { Stage IV } 1 \%\end{array}$ & $\begin{array}{l}\text { Stage I } 4.9 \% \\
\text { Stage IIA } 17.4 \% \\
\text { Stage IIB } 74.1 \% \\
\text { Stage III } 0.4 \% \\
\text { Stage IV } 3.2 \%\end{array}$ \\
\hline Resection status & $\begin{array}{l}\text { R0 } 81 \% \\
\text { R1 } 19 \%\end{array}$ & $\begin{array}{l}\text { R0 39\% } \\
\text { R1 } 61 \%\end{array}$ & $\begin{array}{l}\text { R0 } 59.9 \% \\
\text { R1 } 40.1 \%\end{array}$ \\
\hline Performance status & Karnofsky Performance Status scale $\geq 50 \%$ (median $80 \%$ ) & WHO performance score $\leq 2$ & $\begin{array}{l}\text { WHO performance status } \\
\text { score } \leq 1\end{array}$ \\
\hline Diarrhea & $1 \%$ & $5 \%$ & $18 \%$ \\
\hline Nausea/vomiting & $1.3 \%$ & - & $5.5 \%$ \\
\hline Infection & $0.4 \%$ & $3 \%$ & $3 \%$ \\
\hline Low hemoglobin & $0.6 \%$ & $2 \%$ & $3.4 \%$ \\
\hline Neutropenia & $2.4 \%$ & $38 \%$ & $28.5 \%$ \\
\hline Platelets & $0.8 \%$ & $2 \%$ & $1.3 \%$ \\
\hline Hand-foot syndrome & $0 \%$ & $7 \%$ & $0.4 \%$ \\
\hline Sensory peripheral neuropathy & $0 \%$ & - & $9.3 \%$ \\
\hline \multicolumn{4}{|l|}{ Outcomes } \\
\hline DFS & $13.4 \mathrm{~m}$ & $13.9 \mathrm{~m}$ & $21.6 \mathrm{~m}$ \\
\hline
\end{tabular}

the adjuvant combination of gemcitabine and capecitabine was considered the new standard of care in some subgroups of patients, following resection for pancreatic adenocarcinoma [11].

It is important to mention that in the ESPAC-4 trial, patient characteristics were more representative of the real-world pancreatic cancer population. A large proportion of patients had unfavorable prognostic factors, such as locally advanced or aggressive disease, large tumor size or incomplete removal of the tumor, whereas in the CONKO-001 trial, the majority of patients had undergone an $\mathrm{R} 0$ resection and patients were required to have stage T1-4 N0-1 M0 disease prior to surgery [12]. Overall, patients in ESPAC-4 trial had worst prognostic factors, making the survival results with gemcitabine and capecitabine combination more notable.

However, more adverse events were noted with the combination therapy compared with the monotherapy, especially grade 3 and 4 adverse events such as severe diarrhea (5\% with gemcitabine and capecitabine vs $2 \%$ with gemcitabine alone). The same can be said about grade 3 and 4 neutropenia as it occurred in $38 \%$ of patients who received the combination therapy while it occurred in $24 \%$ of patients receiving monotherapy. Of note, grade 3 and 4 hand-foot syndrome only occurred in the combination regimen, with a frequency of $7 \%$, and was globally manageable [11].

FOLFIRINOX is a regimen based on folinic acid, 5-FU, irinotecan and oxaliplatine. After its approval in the advanced setting, it revolutionized the management of metastatic pancreatic cancer $[13,14]$. Seeing its great success, it was necessary to evaluate this regimen in perioperative and adjuvant setting in early-stage pancreatic cancer.

A new study published in The New England Journal of Medicine in December 2018 tested the efficiency of a modified FOLFIRINOX regimen in resected pancreatic cancer in comparison to gemcitabine. The modified 
regimen, without bolus fluorouracil, was used to decrease the incidence of treatment side effects. The FOLFIRINOX regimen showed better results than gemcitabine monotherapy. In fact, the disease-free survival was prolonged for about 9 months (21.6 vs 12.8 months with gemcitabine). Those results were also significant in the subgroup of patients with poor prognostic factors. This study showed that the OS was also prolonged with FOLFIRINOX (54.4 vs 35 months with gemcitabine). Moreover, the metastasis-free survival and cancer-specific survival were better with FOLFIRINOX than gemcitabine (30.4 vs 17.7 months and not reached vs 36.4 months, respectively) [15]. In this study, patients' characteristics were similar in both groups, except for lymphovascular invasion that was more common in the FOLFIRINOX group.

However, it is noteworthy to mention that grade 3 and 4 adverse events were more frequent with the modified FOLFIRINOX (75.9 vs 52.9\%), including diarrhea, paresthesia, fatigue, sensory peripheral neuropathy, nausea, vomiting, abdominal pain and mucositis. Only thrombocytopenia was more frequent with gemcitabine monotherapy. However, all the toxic effects were reversible (except peripheral neurotoxic effect). In addition, compared with adverse events in the previous PRODIGE trial using unmodified FOLFIRINOX for metastatic disease, grade 3 or 4 neutropenia and diarrhea were reduced with modified FOLFIRINOX by the deletion of bolus fluorouracil and reduction in the irinotecan dose (Table 1) [15].

It might be interesting to find some biomarkers that can predict response to a certain chemotherapy regimen. hENT1 levels can be an efficient predictive biomarker for response to gemcitabine in pancreatic adenocarcinoma. In fact, high hENT1 expression was associated with better response to gemcitabine than low hENT1 expression [16].

In addition, new studies are trying to prove the efficacy of FOLFIRINOX in the neoadjuvant setting. A Phase II clinical trial (NCT01591733) showed that neoadjuvant FOLFIRINOX in localized pancreatic cancer alongside individualized chemoradiotherapy showed better resection margin of the tumor and prolonged PFS and OS [17]. Many clinical trials are studying the efficacy of neoadjuvant therapy in pancreatic cancer. NCT02717091 is a Phase II clinical trial enrolling 50 participants. It is testing the efficacy of neoadjuvant FOLFIRINOX or nab-paclitaxel with gemcitabine in borderline resectable pancreatic cancer.

To sum up, FOLFIRINOX might be the protocol of choice in the adjuvant setting in resectable pancreatic cancer. However, the choice of chemotherapy regimen should be based on tumor and patients' characteristics such as the stage of the disease, age, comorbidities and performance status.

\section{Financial \& competing interests disclosure}

The authors have no relevant affiliations or financial involvement with any organization or entity with a financial interest in or financial conflict with the subject matter or materials discussed in the manuscript. This includes employment, consultancies, honoraria, stock ownership or options, expert testimony, grants or patents received or pending, or royalties.

No writing assistance was utilized in the production of this manuscript.

\section{References}

1. Siegel RL, Miller KD, Jemal A. Cancer statistics, 2018. CA Cancer J. Clin. 68(1), 7-30 (2018).

2. Jemal A, Bray F, Center MM, Ferlay J, Ward E, Forman D. Global cancer statistics. CA Cancer J. Clin. 61(2), 69-90 (2011).

3. McGuigan A, Kelly P, Turkington RC, Jones C, Coleman HG, McCain RS. Pancreatic cancer: a review of clinical diagnosis, epidemiology, treatment and outcomes. World J. Gastroenterol. 24(43), 4846-4861 (2018).

4. Karhu R, Mahlamaki E, Kallioniemi A. Pancreatic adenocarcinoma - genetic portrait from chromosomes to microarrays. Genes Chromosomes Cancer 45(8), 721-730 (2006).

5. Kourie HR, Gharios J, Elkarak F, Antoun J, Ghosn M. Is metastatic pancreatic cancer an untargetable malignancy? World J. Gastrointest. Oncol. 8(3), 297-304 (2016).

6. Miyamoto H, Murakami T, Tsuchida K, Sugino H, Miyake H, Tashiro S. Tumor-stroma interaction of human pancreatic cancer: acquired resistance to anticancer drugs and proliferation regulation is dependent on extracellular matrix proteins. Pancreas 28(1), 38-44 (2004).

7. Oettle H, Post S, Neuhaus $\mathrm{P}$ et al. Adjuvant chemotherapy with gemcitabine vs observation in patients undergoing curative-intent resection of pancreatic cancer: a randomized controlled trial. JAMA 297(3), 267-277 (2007).

8. Oettle H, Neuhaus P, Hochhaus A et al. Adjuvant chemotherapy with gemcitabine and long-term outcomes among patients with resected pancreatic cancer: the CONKO-001 randomized trial. JAMA 310(14), 1473-1481 (2013).

9. Neoptolemos JP, Moore MJ, Cox TF et al. Effect of adjuvant chemotherapy with fluorouracil plus folinic acid or gemcitabine vs observation on survival in patients with resected periampullary adenocarcinoma: the ESPAC-3 periampullary cancer randomized trial. JAMA 308(2), 147-156 (2012). 
10. Maeda A, Boku N, Fukutomi A et al. Randomized Phase III trial of adjuvant chemotherapy with gemcitabine versus S-1 in patients with resected pancreatic cancer: Japan Adjuvant Study Group of Pancreatic Cancer (JASPAC-01). Jpn J. Clin. Oncol. 38(3), 227-229 (2008).

11. Neoptolemos JP, Palmer DH, Ghaneh P et al. Comparison of adjuvant gemcitabine and capecitabine with gemcitabine monotherapy in patients with resected pancreatic cancer (ESPAC-4): a multicentre, open-label, randomised, Phase III trial. Lancet 389(10073), 1011-1024 (2017).

12. Kourie HR, Gharios J, Kattan J. Will gemcitabine monotherapy be dethroned as the adjuvant chemotherapy in pancreatic adenocarcinoma? Future Oncol. 13(3), 197-199 (2017).

13. Conroy T, Desseigne F, Ychou M et al. FOLFIRINOX versus gemcitabine for metastatic pancreatic cancer. N. Engl. J. Med. 364(19), 1817-1825 (2011).

14. Ghosn M, Kourie HR, El Karak F, Hanna C, Antoun J, Nasr D. Optimum chemotherapy in the management of metastatic pancreatic cancer. World J. Gastroenterol. 20(9), 2352-2357 (2014).

15. Conroy T, Hammel P, Hebbar M et al. FOLFIRINOX or gemcitabine as adjuvant therapy for pancreatic cancer. $N$. Engl. J. Med. 379(25), 2395-2406 (2018).

16. Greenhalf W, Ghaneh P, Neoptolemos JP et al. Pancreatic cancer hENT1 expression and survival from gemcitabine in patients from the ESPAC-3 trial. J. Natl Cancer Inst. 106(1), djt347 (2014).

17. Murphy JE, Wo JY, Ryan DP et al. Total neoadjuvant therapy with FOLFIRINOX followed by individualized chemoradiotherapy for borderline resectable pancreatic adenocarcinoma: a Phase 2 clinical trial. JAMA Oncol. 4(7), 963-969 (2018). 